\title{
Material laws and related uncommon phenomena in the electromagnetic response of type-II superconductors in longitudinal geometry
}

\author{
H. S. Ruiz, ${ }^{1, *}$ A. Badía-Majós, ${ }^{1}$ and C. López ${ }^{2}$ \\ ${ }^{1}$ Departamento de Física de la Materia Condensada \\ and Instituto de Ciencia de Materiales de Aragón (ICMA), \\ Universidad de Zaragoza-CSIC, María de Luna 3, E-50018 Zaragoza, Spain \\ ${ }^{2}$ Departamento de Matemáticas, Universidad de Alcalá de Henares, E-28871 Alcalá de Henares, Spain
}

(Dated: March 22, 2018)

Relying on our theoretical approach for the superconducting critical state problem in 3D magnetic field configurations, we present an exhaustive analysis of the electrodynamic response for the socalled longitudinal transport problem in the slab geometry. A wide set of experimental conditions have been considered, including modulation of the applied magnetic field either perpendicular or parallel (longitudinal) to the transport current density. The main objective of our work was to characterize the role of the macroscopic material law that should properly account for the underlying mechanisms of flux cutting and depinning. The intriguing occurrence of negative current patterns and the enhancement of the transport current flow along the center of the superconducting sample are reproduced as a straightforward consequence of the magnetically induced internal anisotropy. Moreover, we show that related to a maximal projection of the current density vector onto the local magnetic field, a maximal transport current density occurs somewhere within the sample. The elusive measurement of the flux cutting threshold (critical value of such parallel component $J_{c||}$ ) is suggested on the basis of local measurements of the transport current density. Finally, we show that a high correlation exists between the evolution of the transport current density and the appearance of paramagnetic peak structures in terms of the applied longitudinal magnetic field.

PACS numbers: 74.25.Vs, 74.25.Ha, 41.20.Z, 02.30.Xx 


\section{INTRODUCTION}

The high interest arisen concerning the problem of magnetic flux depinning in type-II superconductors is markedly associated with its relevance to technological and industrial applications achieving elevated transport currents with no discernible energy dissipation. Thus, the distribution of vortices in a real type-II superconductor is determined by the balance between the electromagnetic driving forces perpendicular to the flux lines, and forces pinning the vortices to material inhomogeneities (lattice defects, impurities, and finite-size effects) ${ }^{1}-3$. Per unit volume, this reads $\mathbf{J} \times \mathbf{B}=\mathbf{F}_{p}\left(\right.$ or $\left.J_{\perp} B=F_{p}\right)$.

On the other hand, it is well-known that various striking phenomena occur when a transport current is applied to a nonideal type-II superconductor under the presence of a longitudinal magnetic field $\underline{\underline{4}-32}$. First, a remarkable enhancement of the critical current density has been observed in a wide number of conventional and high temperature superconducting systems $3,7,22-31$. Second, an intriguing negative longitudinal electric field along the direction of the transport current has been also reported ${ }^{8-21}$. Such a resistive structure in the longitudinal field geometry has been intuitively understood in terms of helical domains, closely connected to the force-free current parallel to flux-lines (recall that the magnetic force per unit volume is given by $\mathbf{J} \times \mathbf{B}) \underline{11,18,21,25}$.

Nevertheless, although several facts suggest that the vortex lattice is arranged in a helical configuration, perhaps close to the force-free arrangement $(\mathbf{J} \| \mathbf{B})$, the arising voltage cannot be straightforwardly described as a critical flux-flow voltage if one is to be consistent with the experimental reports 13,32 . On the other hand, although several proposals have been done in terms of the crossing and recombination of adjacent nonparalell flux lines, the so-called flux line cutting phenomenon has been basically recognized as the physical mechanism by which the longitudinal voltage is produced ${ }^{18}-20,30,31,33-35$. Outstandingly, remarkable numerical and conceptual difficulties related to the implementation of the above picture of the local dynamics for the transport current in realistic longitudinal geometries have lead to unfortunate omissions of the phenomenon in practical calculations related to the applications of type-II materials.

Recently, we have shown that our general critical state theory (Ref. $\underline{4}, 5$ ), is able to describe on a quantitative basis the anomalous features involved in the local electrodynamics of a current carrying superconducting sample subjected to variations of both longitudinal and transverse magnetic fields (Ref. $\underline{\underline{6}}$ ). In brief, our work is developed at a phenomenological level which allows to deal with 3D-magnetic field configurations and nontrivial constraint conditions defining anisotropy effects on the superconducting material law. We have introduced a geometrical description for the constraints on the critical current density in terms of a closed region $\Delta_{\mathbf{r}}$, such that the physically admissible states are given by the condition $\mathbf{J} \in \Delta_{\mathbf{r}}$. When the physical limitations concerning the microscopic phenomena of flux depinning and cutting are considered to be independent, the region $\Delta_{\mathbf{r}}$ becomes a cylinder, with a longitudinal rectangular section of size $2 J_{c \perp} \times 2 J_{c \|}$. This ansatz $z^{4.5}$ can be identified with the so-called double critical state model in type-II superconductivity. Hereafter, a related anisotropy parameter, that we will name after bandwidth $\chi \equiv J_{c \|} / J_{c \perp}$ will be used.

Preliminar work on the intriguing effects associated to the longitudinal transport problem was presented in Ref $\underline{\underline{6}}$. Here, supported by numerical simulations that cover an extensive set of experimental conditions, we put forward a much more complete physical scenario. Thus, we will show that the striking existence of negative flow domains, local and global paramagnetic structures, emergence of peak-like structures in both the critical current density and the longitudinal magnetic moment, as well as the compression of the transport current in type-II superconductors under parallel magnetic fields, are all predicted by our general critical state model. In addition, we shall introduce some ideas that could be applied for the determination of the flux cutting threshold from local measurements of the current density flowing along specific layers of the superconducting sample, as correlated to the behavior of the magnetic moment components.

The paper is organized as follows. In Sec. II] the physical background for the critical state concept in longitudinal geometries under applied 3D magnetic fields is introduced (Sec. II A). Then, the underlying approximations and the variational statement for the longitudinal transport problem in superconducting slabs are both described in detail (Sec. IIB). This encloses the time-discretized description of the magnetic field penetration process, and the local dynamics of the transport current in an arbitrary magnetization process. The theory is then applied for a set of experimental configurations and a collection of material laws as regards the interplay of the flux deppining and cutting mechanisms (Section III). On the one hand, the infinite bandwidth model (or T-state model) with $J_{c \|} \gg J_{c \perp}$ is assumed (Sec. IIIA). As the necessity of including a model with a well defined value for the threshold $J_{c \|}$ will become apparent, in sections ПIIB and IIC a more general description is presented as function of the anisotropy effects on the material law. These effects will be investigated in terms of the range of parameter values: $\chi=1,2,3,4$. The procedure will reveal both local and global properties of the magnetic moment and the transport current flow indicating a possible reconstruction scheme of the underlying material law. Sec. IV is devoted to summarize the main findings of our work. 


\section{LONGITUDINAL TRANSPORT PROBLEM IN THE DOUBLE CRITICAL STATE REGIME}

This section is devoted to introduce the theoretical background that justifies our variational statement as an appropriate tool which allows to implement a wide class of material laws in the investigation of the electromagnetic response of superconducting samples subjected to $3 \mathrm{D}$ magnetic field configurations and transport currents. The characteristic mathematical equations for the critical state problem are introduced in terms of a variational statement that is applied within the infinite slab geometry. The relevance of the parallel and perpendicular critical current limitations will be demonstrated.

\section{A. General statements}

The dynamics of the local magnetic field and transport current profiles in type-II superconductors is commonly obtained within the critical state model framework. In the basic formulation (Bean's model,, $36-38$ ) one considers that the magnetic flux lines, when driven by a macroscopic current density, will either penetrate or exit across the superconducting surface. In more detail, when the driving forces (local value of $\mathbf{J} \times \mathbf{B}$ ) overcome the pinning force in a local section of the superconducting sample, the system of vortices rearranges itself into a new metastable state such that the vortex lattice is pinned again when the equilibrium at the boundary is reestablished. Notice that this physical mechanism relates to the component of $\mathbf{J}$ perpendicular to $\mathbf{B}$. Since the displacement of the flux-lines takes place with high associated resistivity, the system quickly adjusts itself to successive equilibrium states so as to avoid the high resistive losses. On the other hand, reversible energy terms related to the equilibrium properties of the vortex lattice are usually neglected (justified by the range of interest for the local magnetic fields $H_{c 1} \ll H \ll H_{c 2}$ ), and the linear relation $\mathbf{B}=\mu_{0} \mathbf{H}$ is assumed.

For the particular case that the transport current is applied along the direction of the vortex lattice, rotation of vortices is induced by the component of the current density parallel to the magnetic field. Subsequent cross-joining and cutting will occur if the angle between neighboring vortices overcomes some critical value. Thus, in general, for nontrivial configurations, and related to the threshold values for the current density that trigger the displacement of vortex lines in some sense, a critical state may be defined as a given configuration which is able to withstand a critical current defined by $\mathbf{J}_{c}=\mathbf{J}_{c \|}+\mathbf{J}_{c \perp}$, as long as neither threshold $\left(\mathbf{J}_{c \| \mid}\right.$ or $\left.\mathbf{J}_{c \perp}\right)$ is exceeded $\underline{4-6,30,31,35,39}$.

One must mention that, data from a wide number of experiments have shown that in many cases $J_{c \|}$ and $J_{c \perp}$ can be of the same order of magnitude $\underline{-10,12,13,24,40-43}$. Then, in order to explain the accompanying phenomena, both thresholds have to be included in the theory. In these cases, the local current voltage characteristic, connecting the local electric field $\mathbf{E}$ and current density $\mathbf{J}$, is strongly anisotropic with respect to the direction of the magnetic induction B. This fact introduces strong difficulties when trying to find an analytical solution for the critical-state equation $\nabla \times \mathbf{H}=\mathbf{J}_{c}$ (or 0$)$. Our formulation can somehow bypass such difficulties by treating the electric field as a derived quantity that may be obtained a posteriori.

Thus, the critical-state equation can be posed in numerical terms, assuming an evolutionary discretization scheme. Let us suppose that $\mathbf{H}_{l}$ stands for the local magnetic field intensity at the time layer $l \delta t$, and that the current density profiles relate to some magnetic diffusion process that takes place when the local conditions for the material law $J_{\|} \leq J_{c \|}, J_{\perp} \leq J_{c \perp}$ are violated. The successive field penetration profiles within the superconductor may be obtained by the finite-difference expression of Faraday's law,

$$
\mu_{0} \frac{\mathbf{H}_{l+1}-\mathbf{H}_{l}}{\delta t}=-\nabla \times \mathbf{E}=-\nabla \times\left[\rho\left(\nabla \times \mathbf{H}_{l+1}\right)\right] .
$$

Here, $\rho(\mathbf{J})$ plays the role of a nonlinear and nonscalar resistivity that should properly incorporate the physics of the threshold and dissipation mechanisms mentioned above. Notice that the local profile $\mathbf{H}_{l+1}$ can be solved in terms of the previous field distribution $H_{l}$ and the boundary conditions at time layer $(l+1) \delta t$. The initial condition fulfills Ampere's law $\nabla \times \mathbf{H}_{l}=\mathbf{J}_{l}$ as well as $\nabla \cdot \mathbf{H}_{l}=0$ and $\nabla \cdot \mathbf{J}_{l}=0$. One possibility for making the integration of this system affordable is to find an equivalent variational statement. Thus, one can avoid the integration of these set of differential equations by inversion of the Euler-Lagrange equations

$$
\mathbf{J}_{l+1}-\nabla \times \mathbf{H}_{l+1}=0,
$$

and

$$
\nabla \times \mathbf{p}_{l}+\mathbf{H}_{l+1}-\mathbf{H}_{l}=0,
$$

for arbitrary variations of the time-discretized local magnetic field, $\mathbf{H}_{l}$, that plays the role of the unknown. On the other hand, we have introduced the Lagrange multiplier, $\mathbf{p}_{l}$, which can basically identified with the electric field of 
the problem. The related Lagrange density (over whole space) is

$$
L=\frac{1}{2}\left|\mathbf{H}_{l+1}-\mathbf{H}_{l}\right|^{2}+\mathbf{p} \cdot\left(\nabla \times \mathbf{H}_{l+1}-\mathbf{J}_{l+1}\right) .
$$

As indicated above, minimization under the constraint $\nabla \times \mathbf{H}_{l+1}=\mathbf{J}_{l+1}$ (Ampère's law) allows to reduce the number of variables in the statement of the problem, skipping the explicit inclusion of $\mathbf{E}$.

For practical purposes, we stress that the above equations may be transformed so as to get a vector potential formulation by means the condition

$$
\mathbf{H}_{l+1}-\mathbf{H}_{l}=\mu_{0}^{-1} \nabla \times\left(\mathbf{A}_{l+1}-\mathbf{A}_{l}\right) .
$$

Thus, by using standard electromagnetic manipulations, one may show that the functional to be minimized can take the following form in terms of the current density

$$
\begin{aligned}
\mathcal{F}\left[\mathbf{J}_{l+1} \in \Delta_{\mathbf{r}}\right] \equiv & \frac{8 \pi}{\mu_{0}} \int_{\Omega} \Delta \mathbf{A}_{0} \cdot \mathbf{J}_{l+1} d \mathbf{r} \\
& +\iint_{\Omega \times \Omega} \frac{\mathbf{J}_{l+1}^{\prime} \cdot\left[\mathbf{J}_{l+1}-2 \mathbf{J}_{l}\right]}{\left|\mathbf{r}-\mathbf{r}^{\prime}\right|} d \mathbf{r} d \mathbf{r}^{\prime}
\end{aligned}
$$

It must be emphasized that Eq. (6) can be applied for any shape of the superconducting volume $\Omega$ as well as for any general restriction (material law) for the current density $\mathbf{J}_{l+1} \in \Delta_{\mathbf{r}}$. It is also to be noticed that solenoidality $(\nabla \cdot \mathbf{J}=0)$ has to be imposed so as to be consistent with charge conservation in quasi-steady regime.

\section{B. Variational statement in slab geometry}

In this subsection, we derive a specific variational formulation for the longitudinal transport problem in superconducting slabs for eventual 3D-field configurations (Fig. 1), i.e., both in plane and transverse local magnetic field components emerge as derived effects of the external sources (transport currents and/or external magnetic sources).

We shall consider the time evolution of magnetic profiles $\mathbf{H}_{l+1}(z)$ within an infinite superconducting slab (of thickness $2 a$ ), cooled under the assumption of an initial state defined by a uniform vortex lattice perpendicular to the external surfaces, i.e., a constant magnetic field $H_{z 0}$. Then, the constraint for the current density (material law $\mathbf{J} \in \Delta_{\mathbf{r}}$ ) corresponding to the limitations on the parallel and perpendicular directions mentioned above may be visualized by a finite cylinder with its axis parallel to the local magnetic field $H_{z}$ (Fig. 1a). When a transport current is injected along the superconducting slab in the direction of $\mathrm{y}$-axis, a magnetic field component $H_{x}$ appears, inducing a rotation of the critical current region $\Delta_{\mathbf{r}}$ (see Fig. 1 $b$ ). Finally, if a third magnetic field component $H_{y}$ is switched, a new rotation of the region occurs (Fig. 1 ). Notice that, by symmetry, the current density is confined to the $X Y$ plane, i.e.: $\mathbf{J}=\left(J_{x}(z), J_{y}(z), 0\right)$. In particular, this means that in practice one should impose the restriction that $\mathbf{J}$ belongs to the projection of the critical current region onto the plane $\left(\mathbf{J} \in \Delta_{p}\right)$ and that $\nabla \cdot \mathbf{J} \equiv 0$.

At this point, we must mention that for numerical convenience, the material law is not strictly used in the form of a cylinder, but smoothly reshaped as a superellipsoid $\underline{\underline{4}} \underline{\underline{5}}$ by means of the relation

$$
\left(\frac{J_{\|}}{J_{c \|}}\right)^{2 n}+\left(\frac{J_{\perp}}{J_{c \perp}}\right)^{2 n} \leq 1
$$

The critical current region $\Delta_{\mathbf{r}}$ will be characterized in terms of its bandwidth $\chi=\left|J_{c||} / J_{c \perp}\right|$ and the superelliptic index $n$.

We call the readers' attention that two analytical approaches for the slab geometry may be found in the literature for extreme situations. The first one was introduced by Brandt and Mikitik in Ref. $\underline{44}$ for the regime of strong pinning with very weak longitudinal current conditions, i.e., $H_{z}$ must be very high as compared to $H_{x y}(a)\left(\right.$ then $\left.J_{\|} \ll J_{c \|}\right)$. On the other hand, the opposite limit $\left(H_{z} \rightarrow 0\right)$ was recently considered in Ref $\underline{6}$. Here, and based on the numerical resolution of the variational statement, a complete tour along the whole set of values for the perpendicular field will be presented.

In order to simplify the mathematical statements we shall normalize the electrodynamic quantities by defining $\mathbf{h} \equiv \mathbf{H} / J_{c \perp} a, \mathbf{j} \equiv \mathbf{J} / J_{c \perp}$, and $\mathbf{z} \equiv z / a$. In turn, our problem will be described in terms of $N_{s}$ discretized layers, each one characterized by a current density function $\mathbf{j}\left(z_{i}\right)=\mathbf{j}_{x}\left(z_{i}\right)+\mathbf{j}_{y}\left(z_{i}\right)$ distributed along $\left|z_{i}\right| \leq N_{i} a / N_{s}$. For further 
consideration, notice that the value of the transport current assumed in this work, i.e.: penetration to half thickness, imposes the boundary condition $h_{x}(a)=0.5$ (Fig. 2).

In our discretized description, and in terms of Ampere's law $h_{x}\left(z_{i}\right)$ will be evaluated from

$$
h_{x}\left(z_{i}\right)=\delta \sum_{j<i} \frac{2 j_{y}\left(z_{j}\right)-j_{y}\left(z_{i}\right)}{2} .
$$

Similarly, the local profiles for the longitudinal magnetic field component $h_{y}\left(z_{i}\right)$ can be obtained from

$$
h_{y}\left(z_{i}\right)=\delta \sum_{j>i} \frac{2 j_{x}\left(z_{j}\right)-j_{x}\left(z_{i}\right)}{2}
$$

with $\delta \equiv a / N_{s}$ the thickness of each layer over the local plane $z_{i}$.

Then, for the magnetic process sketched in Fig. 1k, Eq. (6) takes the following form in terms of discrete variables

$$
\begin{aligned}
\mathcal{F}(t=l+1) & =\frac{1}{2} \sum_{i, j} I_{i, l+1}^{x} M_{i j}^{x} I_{j, l+1}^{x}-\sum_{i, j} I_{i, l}^{x} M_{i, j}^{x} I_{j, l+1}^{x} \\
& +\frac{1}{2} \sum_{i, j} I_{i, l+1}^{y} M_{i j}^{x} I_{j, l+1}^{y}-\sum_{i, j} I_{i, l}^{y} M_{i, j}^{y} I_{j, l+1}^{y} \\
& +\sum_{i} I_{i, l+1}^{y}(i-1 / 2)\left(h_{l+1}^{y}(a)-h_{l}^{y}(a)\right) .
\end{aligned}
$$

In this expression we have introduced the sheet currents $I_{i, l+1}^{x} \equiv \delta j_{x}\left(z_{i}, t=l+1\right)$ and $I_{i, l+1}^{y} \equiv \delta j_{y}\left(z_{i}, t=l+1\right)$, and their mutual inductance coefficients $M_{i j}^{x, y}$. $\mathcal{F}$ has to be minimized along the different time steps $(l=1,2, \ldots)$ in the magnetic process under consideration. On the other hand, one can show that the parallel and perpendicular projections of the sheet current components are given by

$$
\begin{aligned}
I_{\perp}^{2} & =\left(1-h_{x, i}^{2}\right) I_{x, i}^{2}+\left(1-h_{y, i}^{2}\right) I_{y, i}^{2}-2 h_{x, i} h_{y, i} I_{x, i} I_{y, i} \\
I_{\|}^{2} & =h_{x, i}^{2} I_{x, i}^{2}+h_{y, i}^{2} I_{y, i}^{2}+2 h_{x, i} h_{y, i} I_{x, i} I_{y, i} .
\end{aligned}
$$

These components have to be constrained according to the material law in Eq.(17). Further, for the transport problem, one has to consider the external constraint:

$$
\sum_{i} I_{y, i}=I_{t r}
$$

The theoretical framework becomes closed by the following expressions for the mutual inductance coupling elements 4,5

$$
\begin{array}{r}
M_{i, j}^{x} \equiv 1+2[\min \{i, j\}], \\
M_{i, i}^{x} \equiv 2\left(\frac{1}{4}+i-1\right), \\
M_{i, j}^{y} \equiv 1+2\left[N_{s}-\max \{i, j\}\right], \\
M_{i, i}^{y} \equiv 2\left(\frac{1}{4}+N_{s}-i\right) .
\end{array}
$$

In summary, the magnetic response of the superconductor is characterized by a collection of discretized current elements for the planar sheets $\left(j_{x i}, j_{y i}\right)$ at the time steps $l+1=1,2,3, \ldots$. Then, the magnetic field profiles may be obtained by means of Eqs.(8) and (9), and the sample's magnetic moment per unit area from

$$
\mathbf{M}_{l+1}=\sum_{i} \mathbf{z} \times \mathbf{j}_{i}
$$

where a factor of 2 has been introduced related to the contribution of the U-turns at infinity for each circuit. 


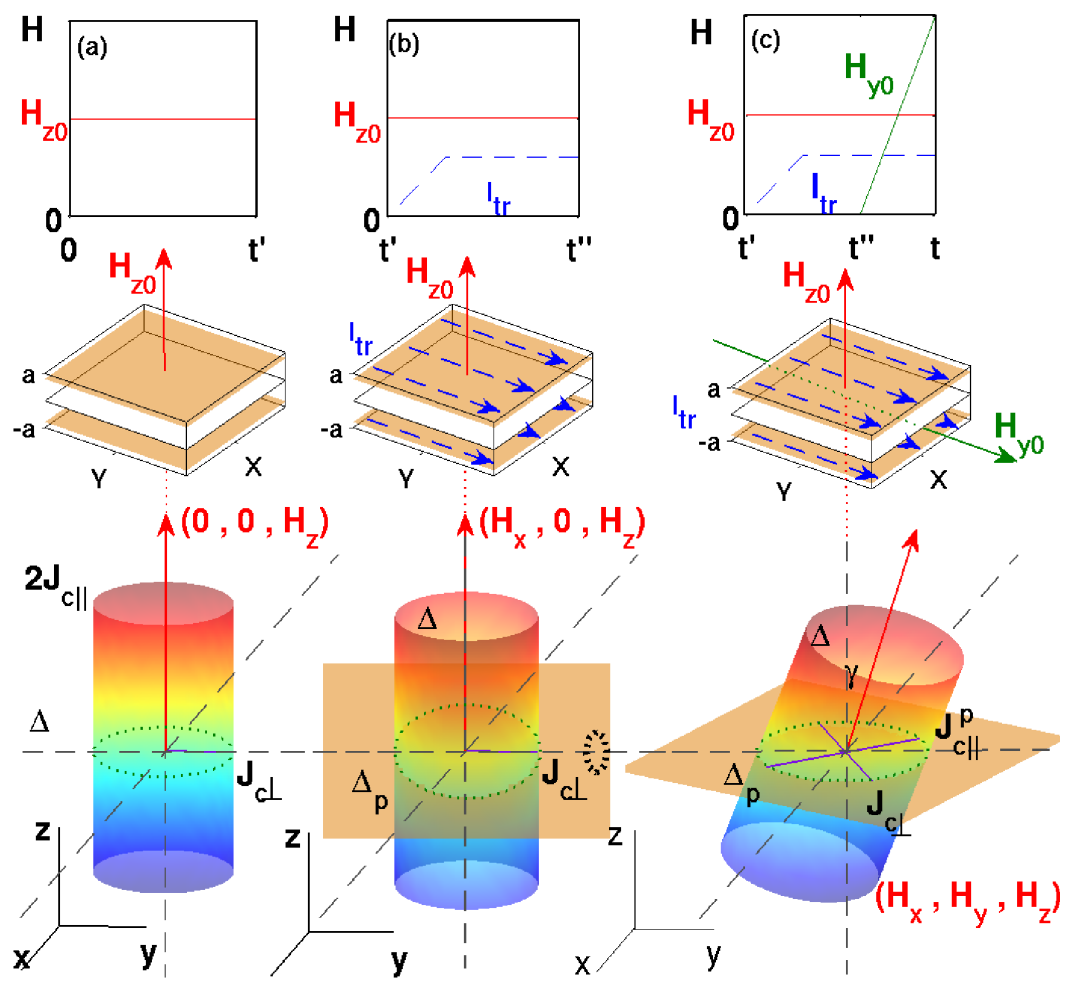

FIG. 1. (Color online) Scheme of the time dependence of the magnetic process considered in this work. (a) Left panel: an external magnetic field $H_{z 0}$ is applied perpendicular to the external surface of a superconducting slab of thickness $2 a$. (b) Middle: the slab is later subjected to a transport current along the $y$-axis. This generates a local magnetic field component $H_{x}$. (c) Right panel: at $t=t^{\prime \prime}$ an external magnetic field $H_{y}$ parallel to the transport of current direction is switched on. The lower row of each panel depicts the region $\Delta$ that constrains the flow of electrical current, as related to the critical state material law. $\Delta$ is a cylinder with axis parallel to the local magnetic field, with radius $J_{c \perp}$ and length $2 J_{c \|}$. The components of $\mathbf{J}$ within the sample's plane are indicated for each case

\section{INFLUENCE OF THE MAGNETIC ANISOTROPY IN THE CRITICAL CURRENT}

In the previous sections we have visualized the double critical state model as a phenomenological approach which can be formulated by means of a variational problem with physical constraints. Here, based on the above mentioned theoretical statements for the longitudinal transport current problem, we will show the theoretical predictions for the magnetization process outlined in Fig. 1 1 as $h_{y}(a) \equiv H_{y 0}$ is increased. In addition, several initial states $h_{z 0}$ will be focused on. Moreover, we shall concentrate on the effect of the flux cutting boundary $\left(j_{c||}\right)$ considering several conditions for the material law. Two extreme cases $(\chi=1$ and $\chi \rightarrow \infty)$ will be considered first (Sections $\amalg$ and (IIB), and the range in between at a second step $(\chi=2,3$, and 4 in Section IIC). Remarkably, our procedure will reveal the fingerprints of the cutting and depinning mechanism, thus being a theoretical pathway for the reconstruction of the material law, represented by the proper region $\Delta_{\mathbf{r}}$.

Henceforth, we shall use the simplified notation $\mathrm{T}$ or $\mathrm{CT} \chi$ as regards to the infinite bandwidth model ( $\mathrm{T}$ by transport) or double critical state model (CT by cutting and transport) with anisotropy $\chi=\left|j_{c||} / j_{c \perp}\right|$.

\section{A. T-states for the longitudinal transport configurations}



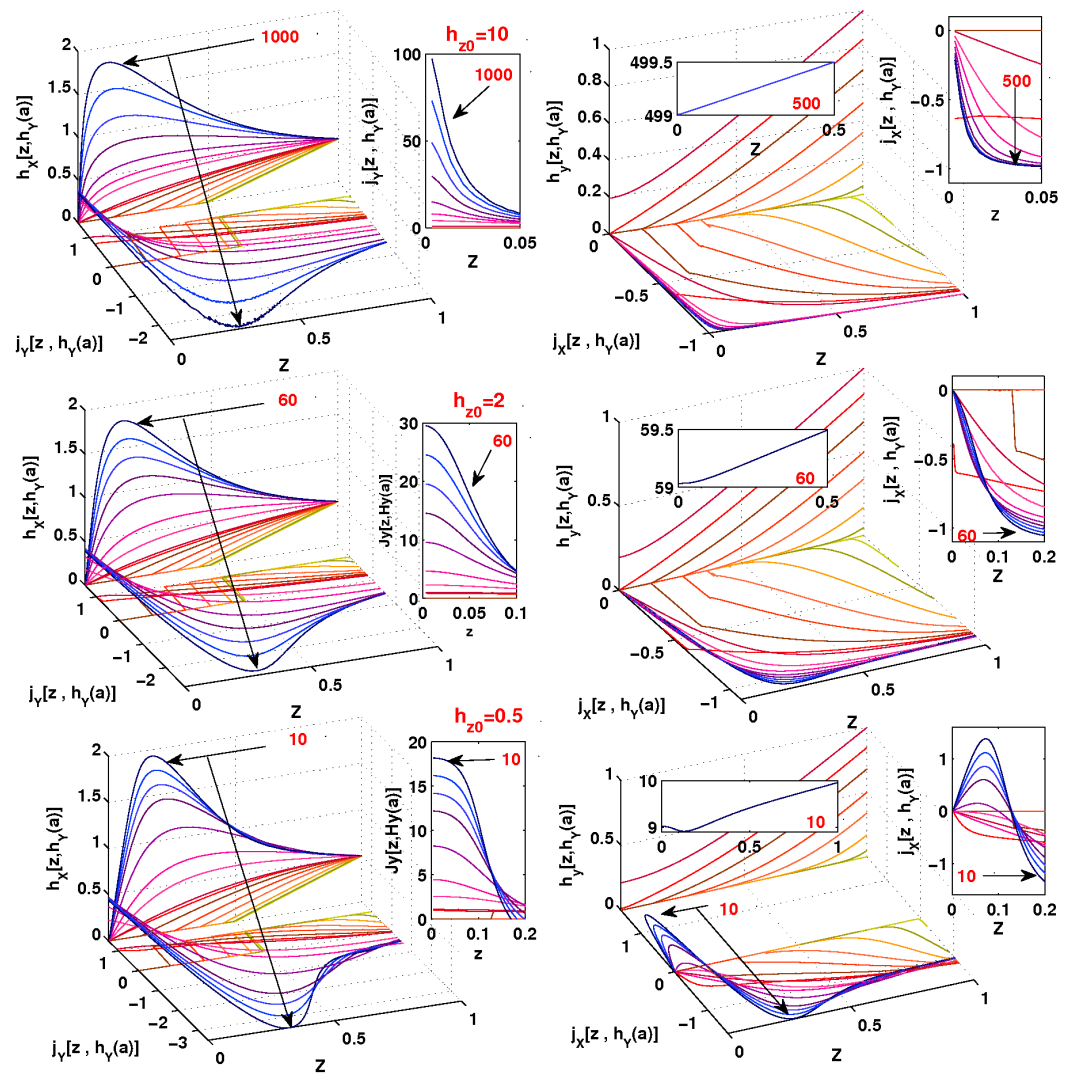

FIG. 2. (Color online) Profiles of the magnetic field components $h_{x}\left[z, h_{y}(a)\right]$ and $h_{y}\left[z, h_{y}(a)\right]$, and the corresponding currentdensity profiles $j_{y}\left[z, h_{y}(a)\right]$ and $j_{x}\left[z, h_{y}(a)\right]$ for the $T$-state model and perpendicular magnetic field components $h_{z 0}=10$ (top), $h_{z 0}=2$ (middle) and $h_{z 0}=0.5$ (bottom). The different curves correspond to the following sets of values for the longitudinal field at the surface: (i) top row: $h_{y}(a)=0.005,0.050,0.170,0.340,0.500,0.680,0.845,1.0,40.0,80.0,150.0,300.0,500.0,750.0$, 1000, (ii) middle row: $h_{y}(a)=0.0050 .050,0.170,0.340,0.500,0.680,0.845,1.0,5.0,10.0,20.0,30.0,40.0,50.0,60.0$, (iii) bottom row: $h_{y}(a)=0.001,0.050,0.170,0.340,0.500,0.680,0.845,1.0,2.0,3.0,5.0,7.0,8.0,9.0,10.0$.

\section{Field and current density penetration profiles}

Fig. 2 shows the magnetic field profiles and the induced currents for three different initial conditions, i.e., $h_{z 0}=10$, $h_{z 0}=2$, and $h_{z 0}=0.5$, all of them under assumption of the T-state model. The initial state for the transport current condition $\left(I_{t r}=J_{c \perp} a / 2\right)$ establishes the initial transport profile $j_{y}\{0 \leq z<a / 2\}=0$ and $j_{y}\{a / 2 \leq z \leq a\}=1$. As the transport current is no longer modified, the condition $h_{x}(a)=0.5$ can be applied in what follows. On the other hand, by symmetry, one has to impose the condition $h_{x}(0)=0$ at the center of the slab.

Then, when the external magnetic field $h_{y}(a)$ is linearly increased from $h_{y}(a)=0$, a current density $j_{x}$ is induced from the superconducting surface as an effect of the Faraday's law. Simultaneously, the local component of the magnetic field $h_{x}(z)$ increases monotonically following two continuous stages fulfilling the aforementioned boundary conditions. First, the superconducting sample is fully penetrated by the transport current when $h_{y}^{\star}(a)=0.845 \pm 0.003$ and eventually, the condition $j_{y}(0)=1$ is reached as soon as $h_{y}(a) \rightarrow 0.860$. We notice that the value of $h_{y}^{\star}(a)$ for the full penetration profile is basically independent of $h_{z 0}$, at least to the numerical precision of our numerical calculations. This agrees with the analytical solution of Ref. .6 . Second, a remarkable enhancement of the transport current density occurs around the center of slab as $h_{y}(a)$ increases over $h_{y}^{\star}(a)$. Furthermore, an eventual negative current density appears shielding the positive transport current around the center of slab. In more detail, notice that the appearance of negative current flow is enhanced when the magnetic component $h_{z 0}$ is decreased (Fig. 3).

Outstandingly, profiles of magnetic field reentry (paramagnetism in the component $h_{y}$ around center of slab) are obtained for $h_{z 0} \lesssim 1$ under relatively low applied magnetic fields $h_{y}(a)$ (see Fig 2).

Another remarkable property is that, for the range of values $h_{z}(0)<h_{y}^{\star}(a)$ negative surface current appears even for the partial penetration regime, e.g., for $h_{z 0}=0.5$ one has $j_{y}(a)<0$ for $h_{y}(a)>0.722$. Recall that, in Ref $\underline{\underline{6}}$ we 

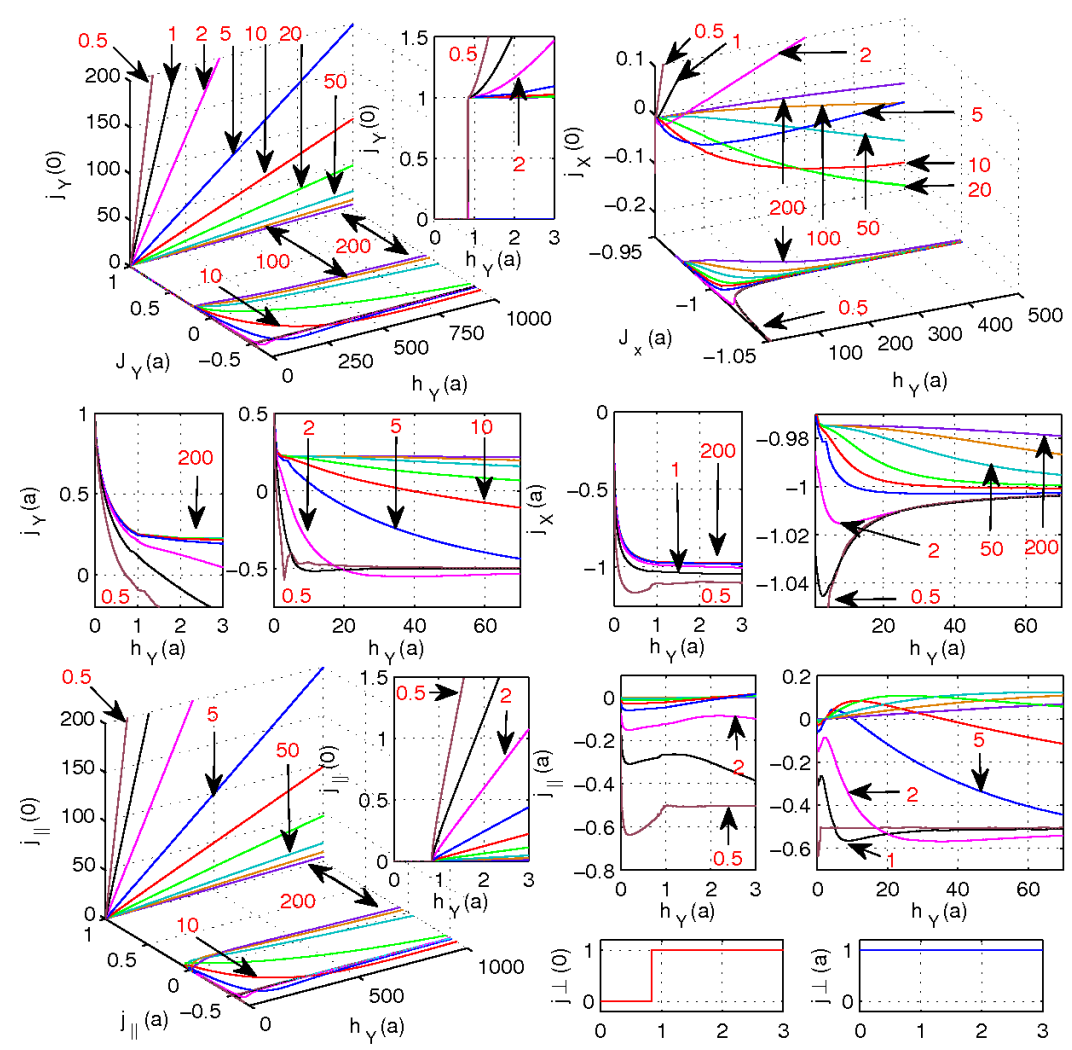

FIG. 3. (Color online) Evolution of the local current density as a function of the applied longitudinal magnetic field $h_{y 0}=h_{y}(a)$ along the central and external sheets of the slab. The results are shown for the T-state model $\left(J_{c \|} \rightarrow \infty \& J_{c \perp}=1.0\right)$. Top: the components $j_{y}$ and $j_{x}$ at $(z=0)$ and $(z=a)$. Middle: details of the above behavior. Bottom: behavior of the parallel and perpendicular components of $\mathbf{j}$ in the same conditions as above. The different curves correspond to the values of the perpendicular magnetic field given by $h_{z 0}=1,2,5,10,20,50,100,200$ (all plots having the same color scale).

have analytically shown that both effects, local paramagnetism and negative current zones are also predicted in the limiting case $h_{z 0}=0$. Along this line, as a general rule, we can conclude that the smaller the value of $h_{z 0}$, the sooner the surface of negative transport current and even paramagnetic local effects appear.

\section{Analysis of the current density behavior}

Fig. [3 displays the evolution of the current density vector as a function of the longitudinal magnetic field $h_{y}(a)$. Note that we focus on the specific values at the superconducting surface $(z=a)$ and at the center of the superconducting $\operatorname{slab}(z=0)$.

In order to understand the physical mechanisms responsible for the observed behavior, it will be useful to consider the following representation of the current density components. We identify the following decomposition of the vector $\mathbf{J}$

$$
\mathbf{J}=\mathbf{J}_{\|}+\mathbf{J}_{\perp \alpha}+\mathbf{J}_{\perp \theta},
$$

that assumes a polar axes representation, with the parallel, azimuth and polar components of $\mathbf{J}$ defined in terms of the magnetic field direction. The following expressions are obtained for such components, when a cartesian coordinate system is used.

(i) The current density parallel to $\hat{\mathbf{H}}$ or so-called cutting current component $J_{\|}$:

$$
J_{\|}=\frac{H_{x} J_{x}+H_{y} J_{y}}{H} .
$$




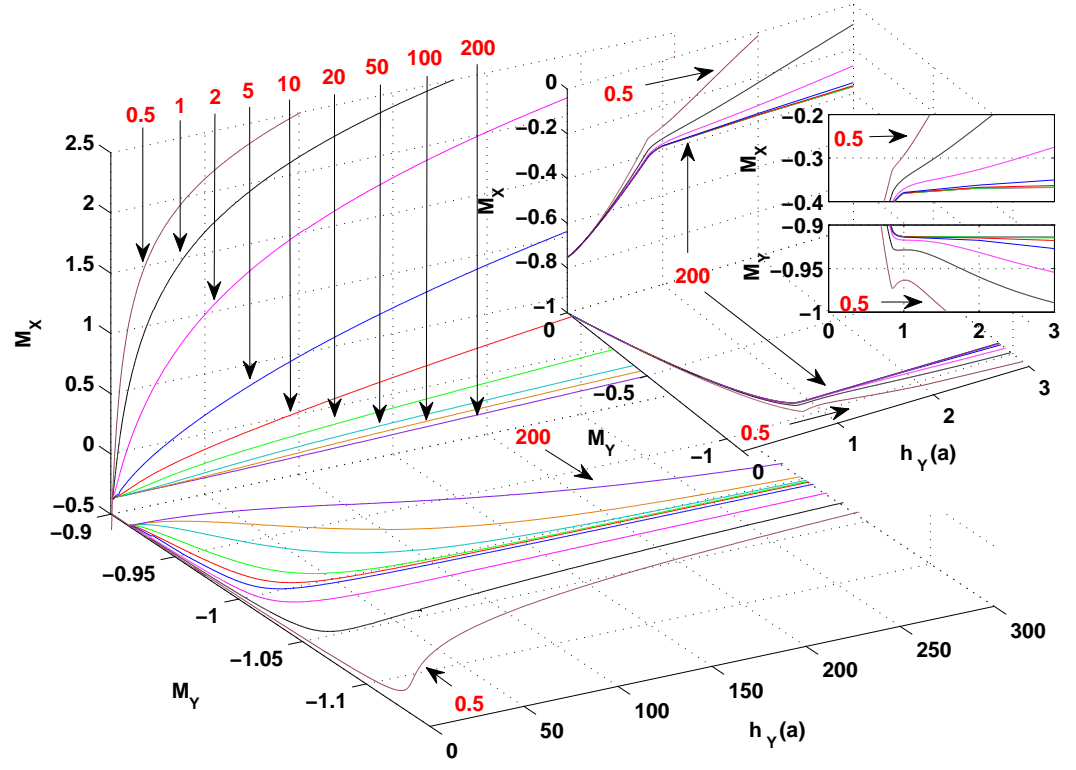

FIG. 4. (Color online) The magnetic moment $\left(M_{x}, M_{y}\right)$ of the slab as a function of the applied magnetic field component $h_{y}(a)$ for the T-state model. The curves are labelled according to the perpendicular magnetic field component $h_{z 0}=1,2,5,10,20,50,100,200$, the same color scale applies to all the plots.

(ii) The component of $\mathbf{J}$ perpendicular to the plane defined by the vectors $\hat{\mathbf{z}}$ and $\hat{\mathbf{H}}$ or so-called azimuthal current component

$$
J_{\perp \alpha}=\frac{-H_{y} J_{x}+H_{x} J_{y}}{\left(H_{x}^{2}+H_{y}^{2}\right)^{1 / 2}}
$$

(iii) the component of $\mathbf{J}$ perpendicular to $\hat{\mathbf{H}}$ and contained in the plane defined by the vectors $\hat{\mathbf{z}}$ and $\hat{\mathbf{H}}$ or so-called polar current component $J_{\perp \theta}$ :

$$
J_{\perp \theta}=\frac{H_{z}\left(H_{x} J_{x}+H_{y} J_{y}\right)}{H\left(H_{x}^{2}+H_{y}^{2}\right)^{1 / 2}} .
$$

Within the T-state model, the behavior of $\mathbf{J}$ is straightforwardly explained: the unbounded parallel current density allows unconstrained rotations for the flux lines as the applied magnetic field increases. In particular, this leads to negative values of $j_{y}(a)$ (slope of $h_{x}(a)$ ), simultaneous to high $j_{y}(0)$ (slope of $h_{x}(0)$ ). We call the readers' attention that negative values of the transport current are favored by smaller and smaller values of the field component perpendicular to the surface of the sample $h_{z 0}$.

A property to be noticed in Fig. 3 is that, at the center of slab the flux line dynamics is mainly governed by the longitudinal transport current density $j_{y}(0)$. The basic idea is that for moderate values of $h_{z}$, when $h_{y}$ increases $j_{y}$ practically becomes $j_{\|}$. As this component is unconstrained, it grows indefinitely at the center.

\section{Magnetic moment of the sample}

Let us now concentrate on the magnetostatic properties by means of the global sample's magnetization curve $\mathbf{M}(\mathbf{H})$. Thus, we have calculated $\mathbf{M}$ as a function of the longitudinal magnetic field $h_{y}(a)$. Fig. 团 displays the magnetic moment components $M_{x}\left(h_{y 0}\right)$ and $M_{y}\left(h_{y 0}\right)$ in units of $J_{c \perp} a^{2}$. Notice first that within the partial penetration regime $\left(h_{y}(a) \leqslant h_{y}^{\star}(a)\right)$ the magnetic moment components are almost independent of the transversal magnetic field $h_{z 0}$ (at least for non small values of this quantity). On the contrary, when $h_{z 0}<1$ and the patterns of negative current even occur before of the full penetrated state, magnetization slightly increases. This is accompanied by faint field reentry effects that are also shown in the figure. Furthermore, as the threshold cutting current $j_{c \|}$ is unbounded for the T-state model, the magnetic moment $M_{x}$ always increases as related to the diverging behavior of $j_{y}(0)$. 
We must emphasize that the unbounded behavior for the parallel current density assumed above (that leads to the prediction of arbitrarily high values of the transport current density) must be physically reconsidered. Thus, the trend of the magnetic moment $M_{x}$ and also the unbounded longitudinal current density $j_{y}$ disagree with the experimental

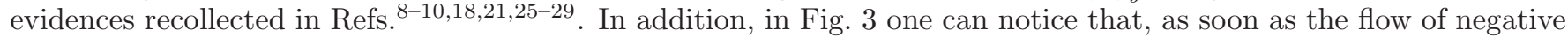
current along the superconducting surface is reached, it never disappears notwithstanding the longitudinal magnetic field remains increasing. By contrast, the disappearance of the patterns of superficial negative current were detected in Refs $\frac{11,14,15,18,21}{1}$. These observations have lead to consider $J_{c \|}$-bounded descriptions as satisfactory solutions of the peculiar phenomena involved on the longitudinal transport current problem ${ }^{8.9}, 11,15$. Rather recent experimental data on high temperature superconductors $\underline{45}$ also indicate that physical bounds are to be considered for both components of the critical current.

On the other hand, the method described in this work suits the necessity of dealing with a physically acceptable description of both local and global issues about the electromagnetic quantities involved on the longitudinal transport current problem $\underline{6}$. More realistic models for the material law are presented below.

\section{B. CT1 states as a general approach}

In this section, we show the results obtained for the square condition given by $\chi \equiv j_{c \|} / j_{c \perp}=1$ (CT1 in what follows). This can be considered as a lower bound for such quantity because the experimental values reported in the literature are typically above unity.

In order to obtain continuity with the T-state results obtained above, the electrodynamic quantities of interest have been proceeded under the same arguments developed in Sec. IIB as regards to the magnetic process shown at Fig. 11.

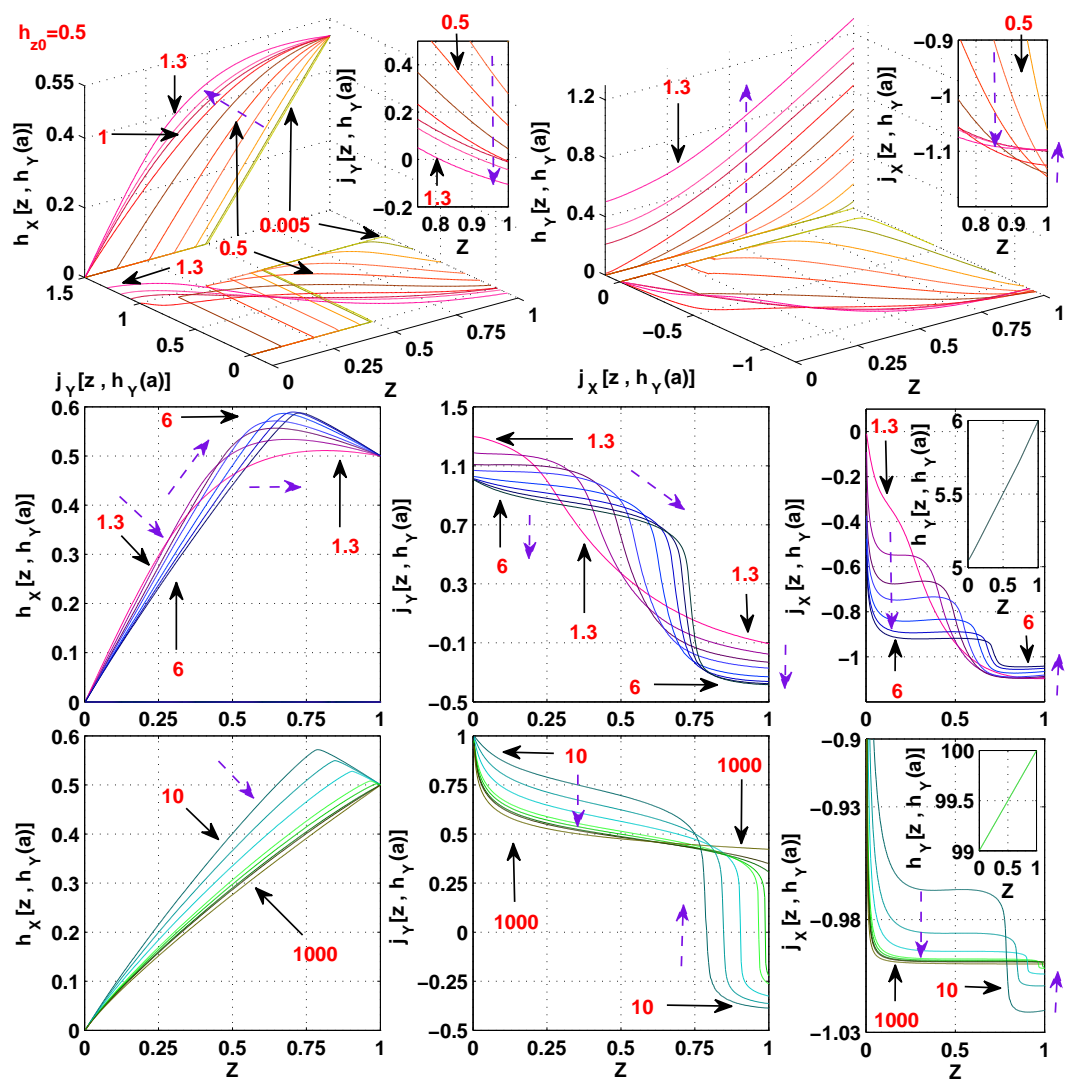

FIG. 5. (Color online) Profiles of the magnetic field components $h_{x}\left[z, h_{y}(a)\right]$ and $h_{y}\left[z, h_{y}(a)\right]$ for a perpendicular field component $h_{z 0}=0.5$. Also included are the corresponding current-density profiles $j_{y}\left[z, h_{y}(a)\right]$ and $j_{x}\left[z, h_{y}(a)\right]$ for the CT1 model. For clarity, the longitudinal magnetic field is applied in three stages: (Top) $h_{y}(a)=0.005,0.050,0.170,0.340,0.500,0.680,0.845,1.0,1.1$, 1.3, (Middle) $h_{y}(a)=1.3,1.6,1.9,2.2,3.0,4.0,5.0,6.0$, and (Bottom) $h_{y}(a)=10,20,40,80,100,125,150,1000$. 


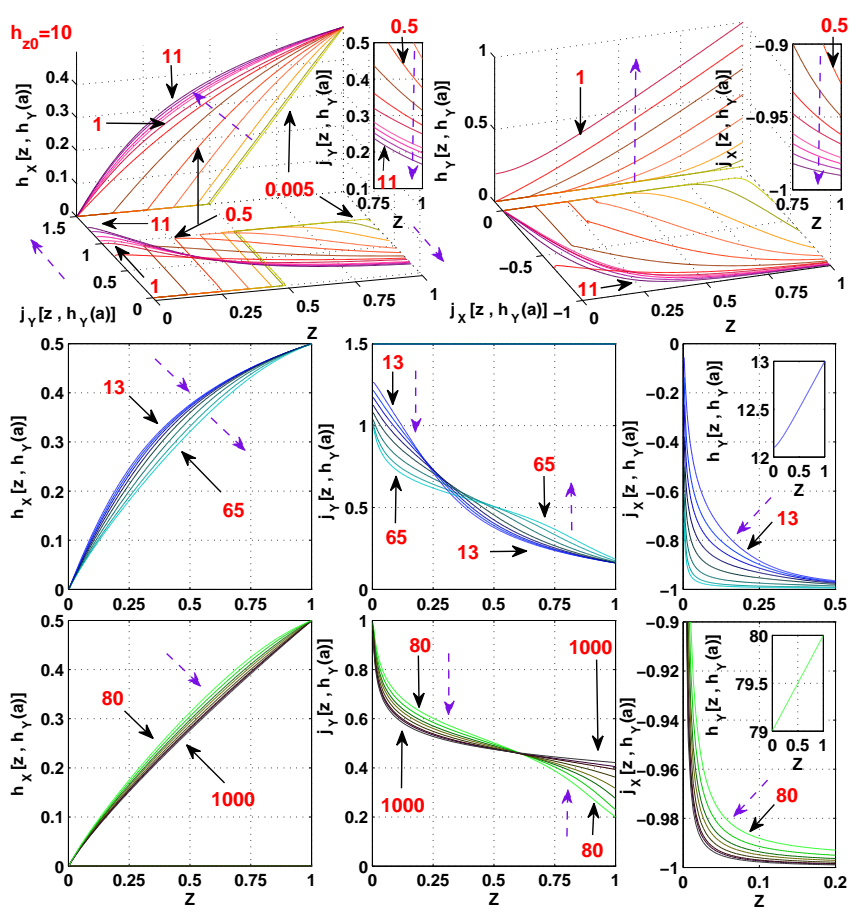

FIG. 6. (Color online) Same as Fig 5 but for $h_{z 0}=10$ and the values of the longitudinal field: (Top) $h_{y}(a)=$ 0.005, 0.050, 0.170, 0.340, 0.500, 0.680, 0.845, 1.0, 4.0, 6.0, 8.0, 11, (Middle) $h_{y}(a)=13,15,17,20,25,35,50,65,($ Bottom $) h_{y}(a)=$ $80,100,125,150,200,300,400,1000$.

On the other hand, with the aim of getting a detailed physical interpretation on how the longitudinal and transverse magnetic fields affect the dynamics of the transport current problem, we show the magnetic penetration profiles for low and high perpendicular fields, i.e., $h_{z 0}=0.5$ (Fig. 5) and $h_{z 0}=10$ (Fig. 6). Eventually, for completeness, the set of initial conditions $h_{z 0}$ is extended in (Fig. 7). It will be shown that the fingerprint of the CT model is identified as a peak effect in the magnetization curves (Fig. 8) caused by the maximal enhancement of the critical current transport density along the central layer.

In order to ease the interpretation of the intricate behavior of the magnetic profiles under CT conditions, we have divided the experimental process in three successive stages as the longitudinal magnetic field component $h_{y}(a)$ is increased, i.e.:

(i) The current density at the center $j_{y}(0)$ increases until a maximum value is obtained (top of Figs. 5 and [6). Notice that the partial penetration regime is included within this stage .

(ii) The minimum value for the longitudinal current density along the superconducting surface is reached (middle row of Figs. 5 and 6).

(iii) The longitudinal current density $j_{y}(a)$ stabilizes around $j_{y}(a) \approx 0.5$ (Bottom of Figs. 5 and 6 ).

\section{Field and current density penetration profiles}

To start with, notice that the trend of the profiles for the partial penetration regime is quite independent of the perpendicular magnetic field $h_{z 0}$ (Figs. [5 and 6). Moreover, the partial penetration regime in which the transport current zone progressively penetrates the sample is practically independent of the magnetic anisotropy of the critical state (compare to Fig. 2). On the other hand, the negative current patterns are also found under the low applied magnetic fields $h_{z 0}<0.5$. However, by contrast to the results within the previous section, recall that for the T-state model the condition $j_{c||} \rightarrow \infty$ allows unbounded values for the longitudinal current $j_{y}$ at the center of the sample. By contrast, for the bounded case, the magnetic anisotropy of the material law $\Delta_{r}$ defines the maximal current density for the critical state regime. In other words, the maximal length of the vector $\mathbf{J}$ within the region $\Delta_{r}$ defines the maximal transport current allowed in the superconductor. 


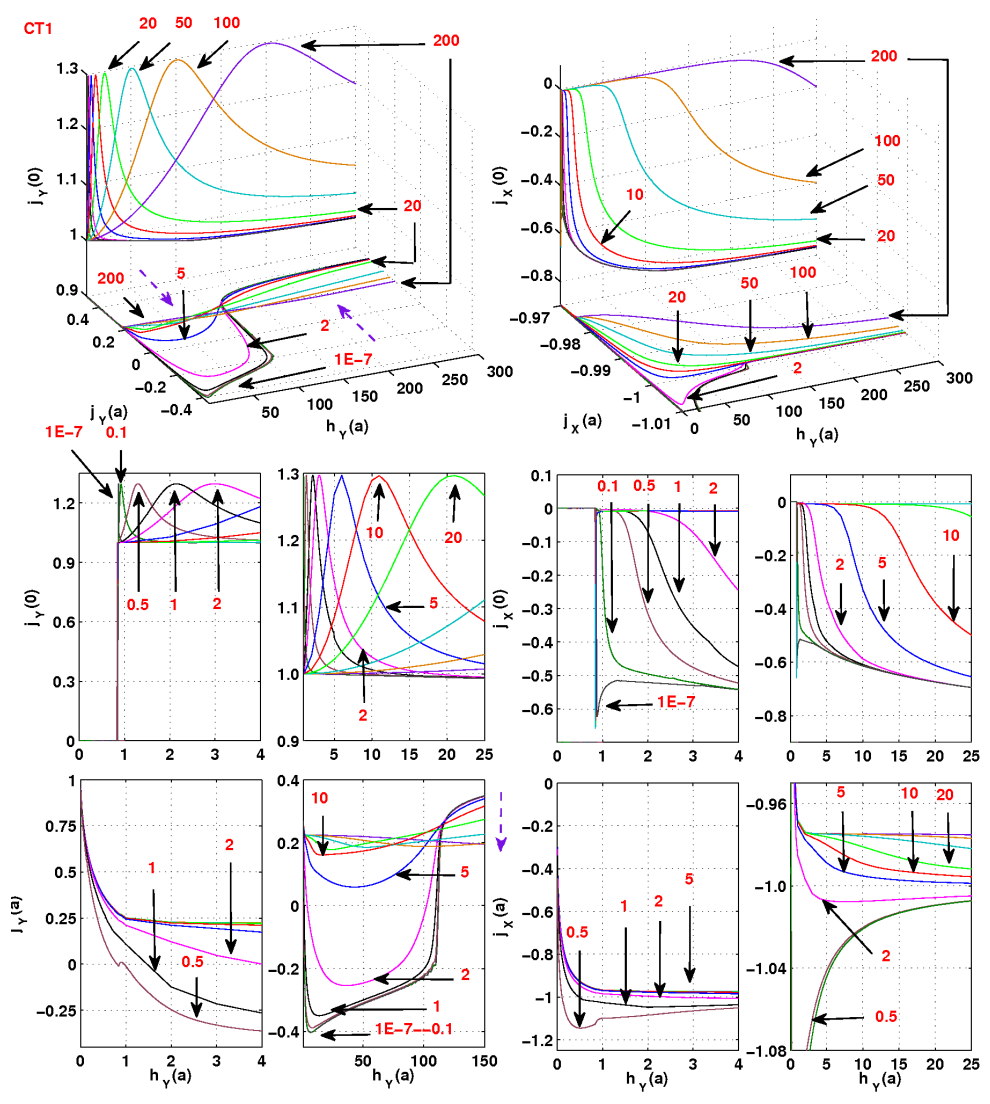

FIG. 7. (Color online) Dynamics of the current density vector for the CT1 model. To the left, we show the patterns of transport current over the central layer $\left(j_{y}(z=0)\right)$ and external layer $\left(j_{y}(z=a)\right)$ of the slab. To the right, we show the variation of the perpendicular component $j_{x}(z=0)$ and $j_{x}(z=a)$. The curves are labelled according to the perpendicular magnetic field component $h_{z 0}=0.1,0.5,1,2,5,10,20,50,100,200$.

Recalling Ref. $\stackrel{4.5}{,}$, the maximal longitudinal transport current density corresponds to the optimal orientation in which the biggest distance within the superelliptic region is obtained, i.e.:

$$
j_{y}^{\max }=\left(1+\chi^{2 n /(n-1)}\right)^{(n-1) / 2 n} .
$$

Note that his equation allows to obtain the maximum value expected for $j_{y}$ in terms of the actual critical state model in use. Thus, for $\chi=1, n=4$ one gets $j_{y}^{\max }=1.3$, a value that can be checked in Figs. [5 and 6 ]

\section{Analysis of the current density behavior}

Complimentary to the field penetration profiles, Fig. 7 shows the dynamics of the main components of the current density along the central and external layers of the superconducting slab in the CT1 condition. Once again, notice that the full penetration regime can be clearly distinguished from the partial penetration regime.

Also, an interesting property to be noticed is that the value $j_{y}^{\max }(0)$ is independent of the applied magnetic field at least as regards the existence of the peak effect in the transport current density (Fig. 7). Thus, the enhancement of the transport current density can be either accelerated or decelerated with the tilt of the applied magnetic field, but in general terms, its maximum directly relates to the limitation introduced by the cutting mechanism. Physically, this means that the role played by the magnetic anisotropy of the material law may be characterized by the influence of the threshold cutting value on the enhancement of the critical current density. This point will be made clearer when material laws with different values of $\chi$ are considered. 

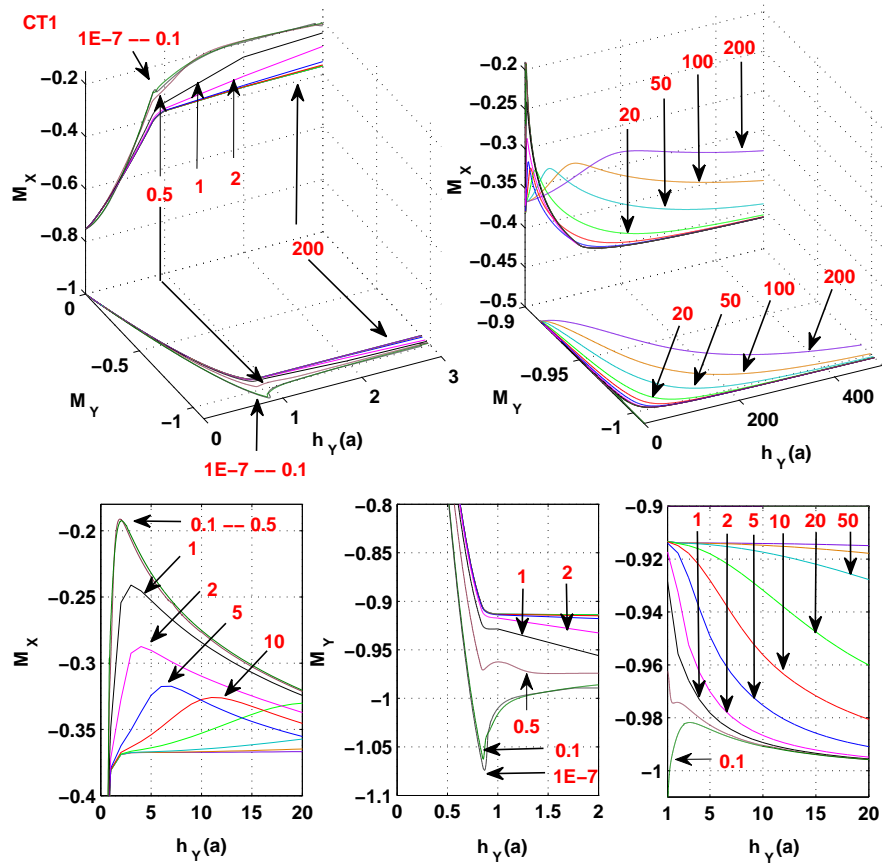

FIG. 8. (Color online) Magnetic moment $\left(M_{x}, M_{y}\right)$ of the slab as a function of the applied magnetic field component $h_{y}(a)$ for the CT1 model. The curves are labelled according to the perpendicular magnetic field component $h_{z 0}$ for each.

\section{Magnetic moment of the sample}

Finally, notice that the limitation introduced by the flux cutting mechanism imposes a maximal compression of the current density within the sample. Thus, the peak effects both for the transport current density $j_{y}$ (Fig. (7) and for the magnetic moment component $M_{x}$ (Fig. 8) are defined by the instant at which the maximal transport current density occurs. Additionally, upon further increasing the longitudinal applied magnetic field component $h_{y}(a)$, the profile $h_{x}(z)$ will be forced to decrease from the central sheet $(z=0)$ towards the external surface $(z=a)$. This reversal generates a local distortion of the longitudinal current density $j_{y}$ in a bow tie shape (see the middle row of Figs. 5 and [6). Likewise, as soon as the profile $\left.j_{\|}(0)=j_{c \|}\right)$ is reached, the magnetic moment $M_{x}$ starts decreasing (Fig. 8) as one can see by comparison of Figs. 7 and 8

\section{CT $\chi$-states: Emergence of the cutting threshold}

The intrinsic interplay between the cutting and depinning mechanisms introduces difficulties when one tries to extract the threshold values for a typical experimental arrangement. Nevertheless, as it was argued in the previous section, several fingerprints of the actual material law can be identified, i.e.: the longitudinal current density along the central and surface superconducting layers, and the behavior of the magnetic moment curves. In order to complement this scenario, we have simulated three additional experiments, defined by the so-called CT2, CT3, and CT4 conditions, in which the threshold value of the cutting current density is varied relative to the depinning limit $(\chi=2,3,4$ respectively).

\section{Field and current density penetration profiles}

The behavior of the local electrodynamic quantities is displayed in terms of the aforementioned three stages. In order to allow comparison, the main features displayed in Figs. 5 and 6 for the CT1 condition with $h_{z 0}=0.5$ and 10, are also shown in Figs. 9 and 10 under the conditions CT2, CT3 and CT4. Several peculiarities are to be noted. To start with, the emergence of negative states for the transport current density close to the external surface of the 

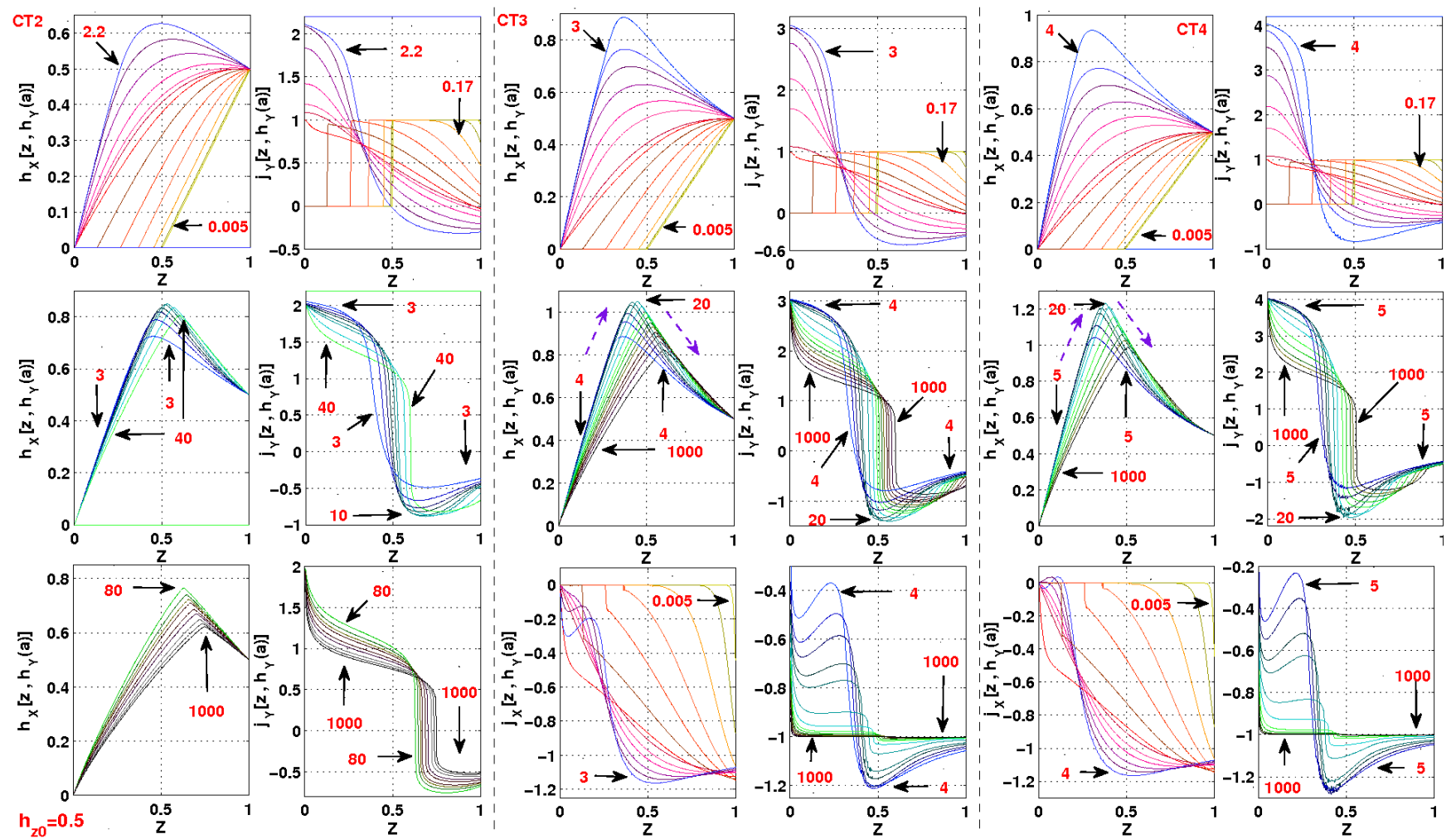

FIG. 9. (Color online) Profiles of $h_{x}\left[z, h_{y}(a)\right]$ and $j_{y}\left[z, h_{y}(a)\right]$ for the 1st (top) and 2nd (middle) stages of the magnetic dynamics described in CT2, CT3, and CT4 cases (see text) all under a field $h_{z 0}=0.5$. The 3rd stage is only defined for the CT2 case (bottom). $h_{y}(a)$ is as follows. CT2: 1st stage $h_{y}(a)=$ $0.005,0.050,0.170,0.340,0.500,0.680,0.845,1.0,1.1,1.3,1.6,1.9,2.2$, 2nd stage $h_{y}(a)=3,4,5,6,8,10,20,40$, and 3 rd stage $h_{y}(a)=80,120,160,200,300,400,600,800,1000$. CT3: 1st stage $h_{y}(a)=0.005,0.050,0.170,0.340,0.500,0.680,0.845,1.0,1.5$, $1.8,2.2,2.6,3.0$, and 2nd stage $h_{y}(a)=4,5,6,8,15,20,40,70,100,150,200,300,400,600,1000 . \quad$ CT4: 1st stage $h_{y}(a)=0.005,0.050,0.170,0.340,0.500,0.680,0.845,1.0,1.5, \quad 1.8,2.2,2.6,3.0,4.0, \quad$ and $\quad 2$ nd $\quad$ stage $h_{y}(a) \quad=$ $5,6,8,15,20,40,100,200,400,600,1000$.

superconducting sample will be more evident either when $h_{z 0}$ is reduced and/or the current density anisotropy factor $\chi$ is increased.

\section{Analysis of the current density behavior}

In order to confirm the above interpretation, we show the magnetic dynamics of the longitudinal current density $j_{y}$, the cutting current component $j_{\|}$(Fig. 11) for the conditions CT2, CT3, and CT4. We have taken a wide set of values for the perpendicular field component $\left(h_{z 0}\right)$. On the one hand, as regards the sample's surface, Fig. 11] shows that the longitudinal current density $j_{y}(a)$ does not display significative differences when one has $\chi \geq 2$ (see also Fig. 3 for $\chi=\infty$ ). Thus, the disappearance of the negative current flow along the external superconducting surface does not occur despite a very high applied magnetic field has been considered $\left(h_{y}(a)=1000\right)$. On the other hand, it is important to notice that the patterns of the parallel current density along the superconducting surface $\left(j_{\|}(a)\right)$ are almost indistinguishable as soon as the condition $\chi \geq 2$ (CT2) is reached (upper half of Fig. 11). This implies that for an accurate picture of the parallel critical current, surface properties do not provide a useful information.

However, outstandingly, Fig. 11 shows that the threshold value for the cutting current density can be estimated from the experimental measurement of the transport current density along the central sheet of the superconducting sample. Notice further that, regardless of the experimental conditions $\left(h_{z 0}, h_{y}(a)\right)$ and also for different bandwidths $\chi$ no significant change occurs in the parallel current density around the central sheet of the sample (lower half of Fig. 11). 

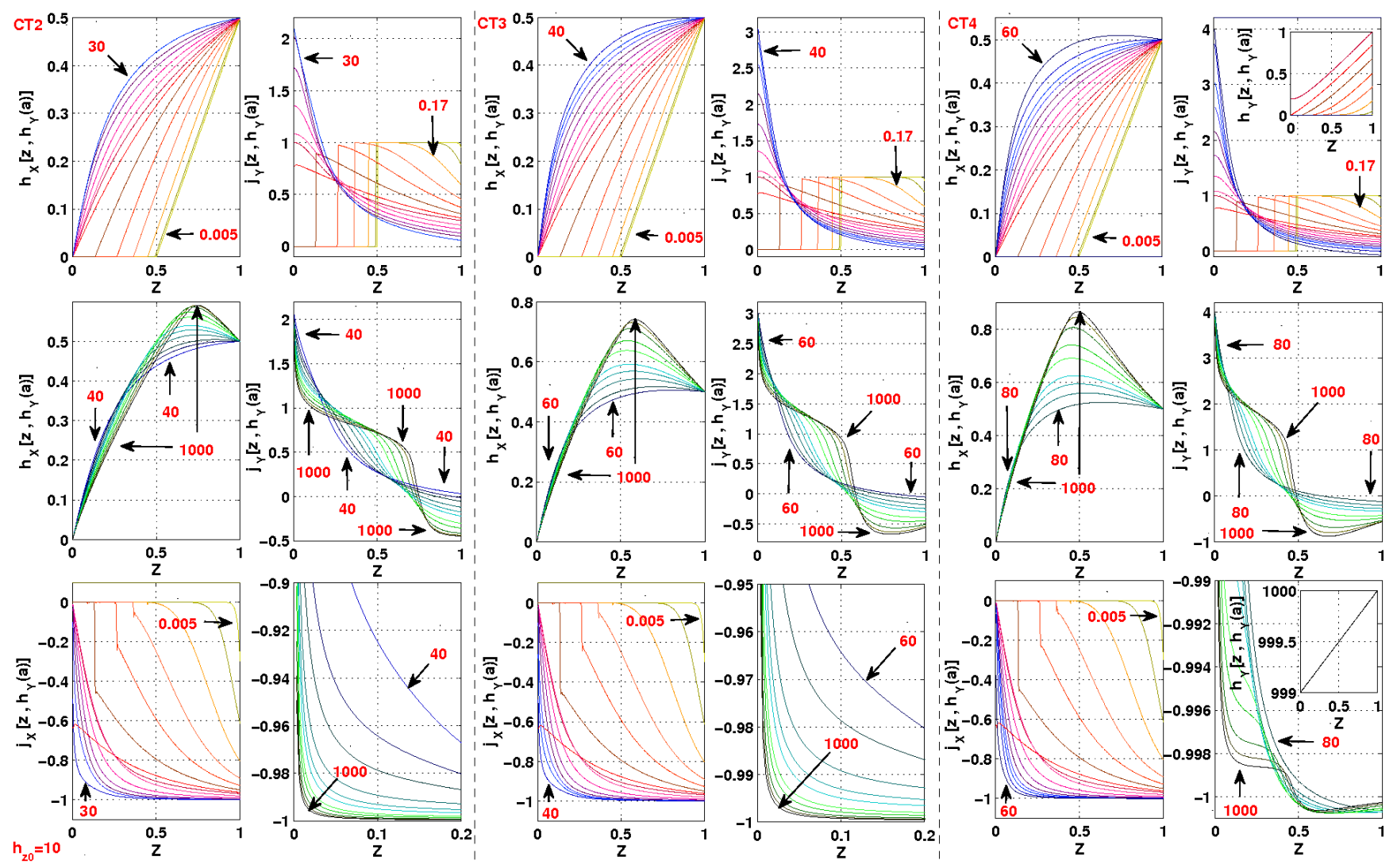

FIG. 10. (Color online) Similar to Fig. 9] but for a perpendicular magnetic field component $h_{z 0}=10$. The curves are labelled as follows. (CT2 - left panel): 1st stage $h_{y}(a)=0.005,0.050,0.170,0.340,0.500,0.680,0.845,1.0,5.0$, $10,15,20,30$, and 2nd stage $h_{y}(a)=40,60,80,120,160,200,300,400,600,800,1000$. (CT3-middle panel): $\quad 1$ st stage $h_{y}(a)=0.005,0.050,0.170,0.340,0.500,0.680,0.845,1.0,5.0, \quad 10,15,20,25,30,40, \quad$ and 2nd stage $h_{y}(a)=60,80,120,160,200,300,400,600,800,1000 . \quad$ (CT4 $\quad$ right panel): 1st stage $h_{y}(a)=0.005,0.050,0.170,0.340,0.500,0.680,0.845,1.0,5.0, \quad 10,15,20,35,30,40,60, \quad$ and 2 nd $\quad$ stage $h_{y}(a) \quad=$ $80,120,160,200,300,400,600,800,1000$.

\section{Magnetic moment of the sample}

The peak effects observed both in the local transport current density $j_{y}(0)$ (lower half of Fig. 11), and on the global magnetic moment $M_{x}$ (Fig. 12), are predicted to appear subsequent to the maximal longitudinal transport current density $j_{y}^{\max }$ (Eq. [19).

Furthermore, one additional feature is to be noted: the interval between the instant at which the maximal transport current condition is reached $\left(j_{y}(0)=j_{y}^{\max }\right)$, and the instant at which the slope of the magnetic moment $M_{x}$ changes sign, could be assumed as transient or stabilization period required for an accurate determination of the value $j_{c \mid l}$ when measurements are performed in terms of the applied longitudinal field $h_{y}$. Apparently, this transient increases with the value of the perpendicular field $h_{z 0}$. From this point on, $j_{y}(0)$ may be basically identified with $j_{c \|}$.

\section{CONCLUSIONS}

Despite of extensive experimental and theoretical studies about the electrodynamic response of type-II superconductors in longitudinal geometries, much uncertainties remain about the interaction between flux depinning and cutting mechanisms, and their influence in such striking observations as the appearance of negative transport current flow, the enhancement of the critical transport current density, and the observation of peak effects on the magnetization curves. In this article, and based on the application of our general critical state theory, we have reproduced theoretically the existence of negative flow domains, local and global paramagnetic structures, emergence of peak-like 

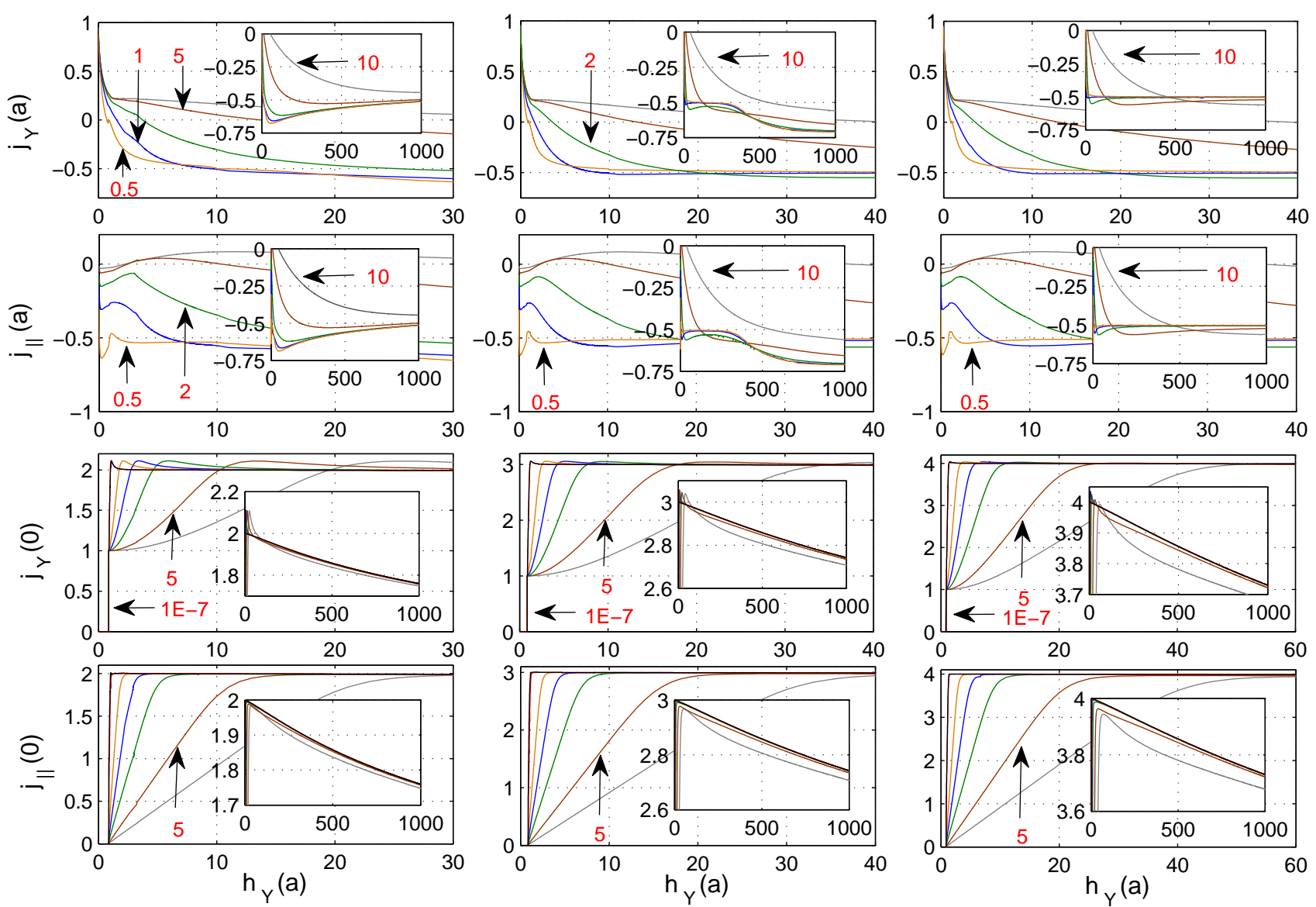

FIG. 11. (Color online) Dynamics of the current density vector for the CT2, CT3 and CT4 models. In the upper panel, we show the patterns of transport current along the central $\left(j_{y}(z=0)\right)$ and surface layers $\left(j_{y}(z=a)\right)$ of the slab. The curves are labelled according to the perpendicular magnetic field component $h_{z 0}=1 E-7,0.5,1,2,5,10$

structures in the longitudinal magnetic moment, as well as the compression of the transport current density for a wide number of experimental conditions.

The longitudinal transport problem in superconducting slab geometry has been studied as follows: we have considered a superconducting slab lying at the $x y$ plane and subjected to a transport current density along the $y$ direction. The slab is assumed to be penetrated by a uniform vortex array along the $z$ direction, so that the local current density along the thickness of the sample is entirely governed by the depinning component $J_{\perp}$, perpendicular to the local magnetic field. Subsequently, a magnetic field source parallel to the transport current direction is switched on. Then, the experimental conditions have been modulated through the value of the external magnetic field $H_{y 0}$. The dynamical behavior of the transport current density is shown to rely on the interaction between the cutting and depinning mechanisms. Moreover, the intensity of the inherent effects has been shown to depend on the perpendicular component $H_{z 0}$, being more prominent as this quantity is reduced. By means of our general critical state theory that allows to modulate the influence of the different physical effects, we have been able to show that the peak structures observed in the magnetization curves and the patterns of the transport current along the central section of a superconducting sample are both directly associated with the local structure of the vortex lattice. Such dependence may become more pronounced as the extrinsic pinning of the material is reduced, in favor of the flux cutting interactions. The same conclusion was pointed out from the experimental measurements of Blamire et. al (Ref $\underline{7,23}$ ) for high critical temperature and conventional superconductors. By using a theory that depends on two parameters $\left(\chi \equiv J_{c \|} / J_{c \perp}\right.$ and $n$ ) accounting respectively for the intrinsic material anisotropy and for the smoothness of the $J_{\|}\left(J_{\perp}\right)$ law, here, we have quantitatively investigated the influence of the flux cutting mechanism. It has been done by comparing the so-called square model $(\chi=1)$, the T-state model $(\chi \rightarrow \infty)$, and the double critical state conditions CT $\chi$ with $\chi=2$, $\chi=3$, and $\chi=4$, all of them with the smoothing index $n=4$.

From our theoretical framework we obtain that the isotropic model (circular region: $\chi=1, n=1$ ) does neither 

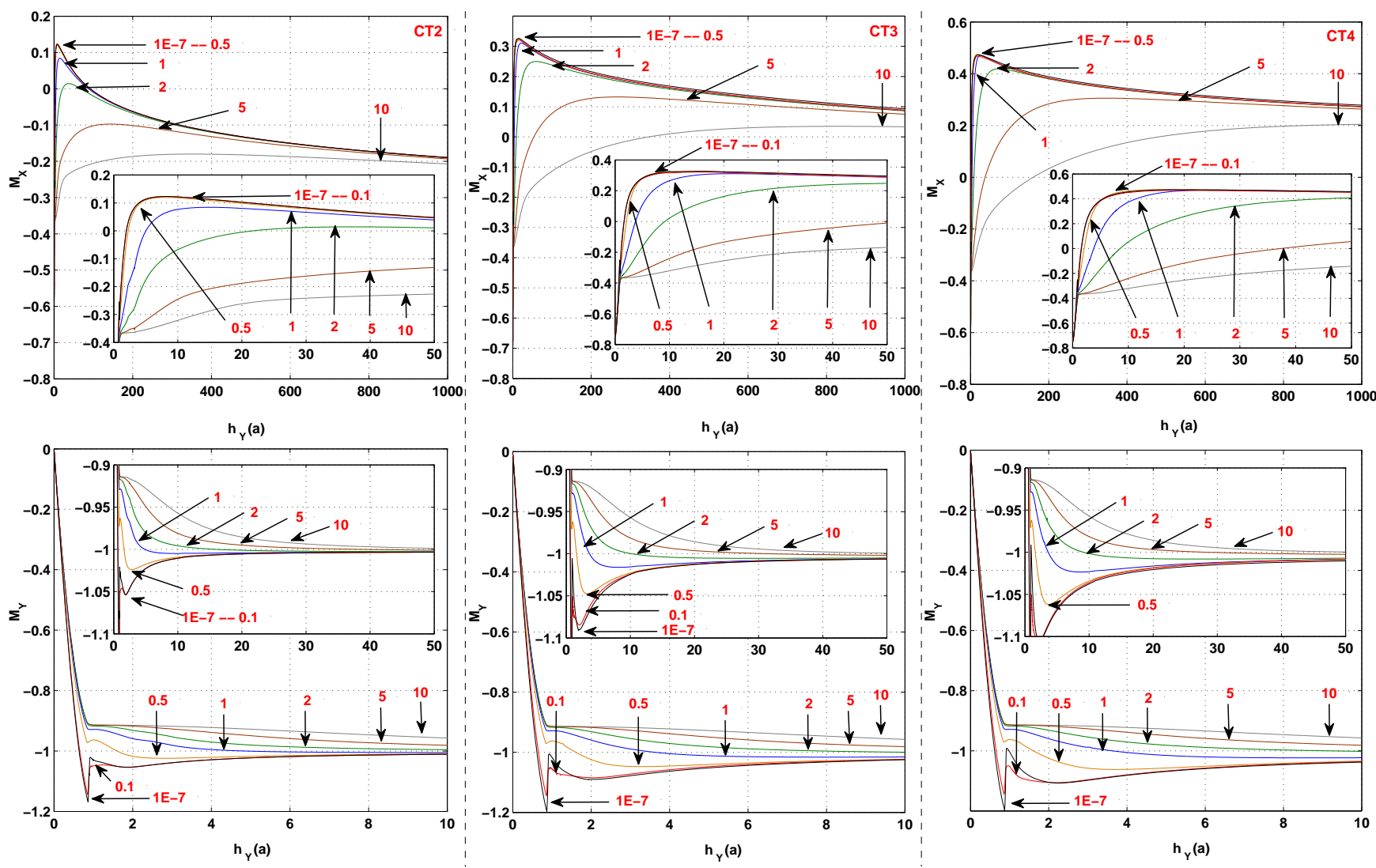

FIG. 12. (Color online) The magnetic moment $\left(M_{x}, M_{y}\right)$ of the slab as a function of the applied magnetic field component $h_{y}(a)$ for the CT2, CT3, and CT4 smooth critical state models. The curves are labelled according to the perpendicular magnetic field component $h_{z 0}$ in correspondence at the Fig 11

predict the appearance of negative current patterns nor the peak effects in the magnetic moment curves. However, as long as a clear distinction between the depinning and the cutting components of $\mathbf{J}$ is allowed, several remarkable facts can be explained.

Going into detail, when the cutting threshold is high $\left(J_{c \|} \gg J_{c \perp}\right.$ or $\left.\chi \gg 1\right)$ the emergence of negative current patterns is ensured because unbounded parallel current density allows unconstrained rotations for the flux lines as the longitudinal magnetic field increases. Thus, under a range of conditions, the peak effects in the magnetic moment and a modulation of the negative surface currents are predicted.

Concentrating on the local properties within the sample, a clear independence of the field penetration profiles relative to the anisotropy level of the material law has been obtained for the partial penetration regime. On the other hand, as soon as the full penetration state is reached, noticeable effects of the magnetic anisotropy law are predicted both within the central and external layers of the superconducting sample. Thus, according to our results (see Fig [11) the elusive parallel critical current density parameter $J_{c||}$ can be obtained from the local measurement of the transport current density along the central layer of the superconducting sample.

\section{ACKNOWLEDGEMENT}

This work was supported by Spanish CICyT and FEDER program (project MAT2008-05983-C03-01) and by the DGA grant T12/2011. H. S. Ruiz acknowledges the financial support provided by the Spanish CSIC JAE-program. 


\section{REFERENCES}

* hsruizr@unizar.es

1 Rosenstein B, Shapiro I and Shapiro B Ya 2010 Phys. Rev. B 81064507

${ }^{2}$ Voloshin I F , Fisher L M and Yampol'skiǔ V A 2010 Low Temp. Phys. 3639

3 Sánchez A, Navau C, Del-Valle N, Chen D X and Clem J R 2010 Appl. Phys. Lett. 96072510

4 Badía-Majós A, López C and Ruiz H S 2009 Phys. Rev. B 80144509

5 Ruiz H S and Badía-Majós A 2010 Supercond. Sci. Technol. 23105007

6 Ruiz H S, López C and Badía-Majós A 2011 Phys. Rev. B 83014506

7 Blamire M G, Marrows C H, Stelmashenko N A and Evetts J E 2003 Phys. Rev. B 67014508

8 LeBlanc M A R and Çelebi S 2003 Supercond. Sci. Technol. 16329

${ }^{9}$ Çelebi S and LeBlanc M A R 2002 Studies of High-Temperature Superconductors vol 42 (New York: Nova Science) p 169

10 Voloshin I F, Kalinov A V, Fisher L M, Derevyanko S A and Yampol'skiǔ V A 2001 JETP Lett. 73285

11 Matsushita T, Shimogawa A and Asano M 1998 Physica C 298115

12 LeBlanc M A R, Çelebi S, Wang S X and Plechácêk V 1993 Phys. Rev. Lett. 713367

13 LeBlanc M A R, LeBlanc D, Golebiowski A and Fillion G 1991 Phys. Rev. Lett. 663309

14 Voloshin I F, II'in N V, Makarov N M, Fisher L M and Yampol'skiü V A 1991 JETP Lett. 53116

15 Matsushita T and Irie F 1985 J. Phys. Soc. Japan 541066

16 Cave J R and Evetts J E 1978 Phil. Mag. B 37111

17 Wamsley D G and Timms W E 1977 J. Phys. F 72373

18 Esaki T and Irie F 1976 J. Phys. Soc. Japan 40382

19 Wamsley D G 1972 J. Phys. F 2510

${ }^{20}$ London H and Wamsley D G 1968 Proc. 11th Int. Conf. Low Temp. Phys. (St. Andrews) p 879

21 LeBlanc M A R 1966 Phys. Rev. 143220

22 Watanabe K, Yamane H, Kobayashi N, Hirai T and Muto Y 1992 Studies of High-Temperature Superconductors vol 8 (New York: Nova Science) p 107

23 Blamire M G and Evetts J E 1986 Phys. Rev. B (Rapid Communications) 335131

24 Boyer R, Fillion G and LeBlanc M A R 1980 J. Appl. Phys. 511692

${ }^{25}$ Gauthier R, LeBlanc M A R and Belanger B C 1974 it Proceedings of Low Temperature Physics-LT13 vol 3 (New York: Plenum) p 241

${ }^{26}$ Karasik V R and Vereshchagin V G 1970 Zh. Eksp. Teor. Fiz. 5936 [1971 Sov. Phys.-JETP 32 20]

27 Sugahara M 1970 Japan. J. Appl. Phys. 9625

28 Taylor H F 1967 Appl. Phys. Lett. 11169

29 Sekula S T, Boom R W and Bergeron C J 1963 Appl. Phys. Lett. 2102

30 Clem J R 1980 J. Low Temp. Phys. 38 353, and references quoted therein.

31 Clem J R and Yeh S 1980 J. Low Temp. Phys. 39 173, and references quoted therein.

${ }^{32}$ Nakayama Y 1972 Proceedings of the 4th International Cryogenic Engineering Conference (Guildford: IPC Science and Technology Press) p 133

33 Brandt E H 1980 J. Low. Temp. Phys. 3941

${ }^{34}$ Campbell A M and Evetts J E 1972 Adv. Phys. 21199

35 Clem J R 1975 Phys. Lett. 54A 452

36 Bean C P 1962 Phys. Rev. Lett. 8250

37 Bean C P 1964 Rev. Mod. Phys. 3631

38 Bean C P 1970 J. Appl. Phys. 412482

39 Clem J R 1977 Phys. Rev. Lett. 381425

40 Boyer R and LeBlanc M A R 1977 Solid State Commun. 24261

41 Fillion G, Gauthier R and LeBlanc M A R 1979 Phys. Rev. Lett. 4386

42 Cave J R and LeBlanc M A R 1982 J. Appl. Phys. 531631

43 LeBlanc M A R and Lorrain J R 1984 J. Appl. Phys. 554035

44 Brandt E H and Mikitik G P 2007 Phys. Rev. B 76064526

45 Clem J R, Weigand M, Durrell J H and Campbell A M 2011 Supercond. Sci. Technol. 24062002 